\title{
Las regulaciones iusprivatistas para el contrato de trabajo internacional como forma de protección al migrante: apuntes desde Cuba*
}

\author{
The iusprivatists for the international \\ employment contract as a form of protection \\ for migrants: Notes from Cuba
}

Odette Martínez Pérez*

\begin{abstract}
RESUMEN
En los últimos años se han producido cambios notables en el contexto económico internacional. Se abre camino un mundo donde las economías cada vez están más integradas. Los avances tecnológicos y las reformas aperturistas incrementan el crecimiento del comercio y los flujos de inversión. Todo ello se traduce en un incremento de la migración, que impacta a las sociedades en importantes campos, como el laboral. Por tanto, han de buscarse mecanismos convencionales y otros cuya función principal no es la de tutela, para la protección de los migrantes. Es el caso del contrato de trabajo internacional.
\end{abstract}

PALABRAS CLAVE: Migración, trabajo, contrato, Cuba, derecho.

\begin{abstract}
In recent years there have been notable changes in the international economic scenery. It is opening to a world that is making its way to economies that are increasingly integrated. Technological advances and opening reforms increase trade growth and investment flows. All this translates into an increase in migration, which impacts societies in important fields, such as labor. Therefore, conventional mechanisms are to be found and others whose main function is not of guardianship should be sought for the protection of migrants. This is the case of The International Employment Contract.
\end{abstract}

KEY WORDS: Migration, work contract, Cuba, Law.

\footnotetext{
* Artículo de reflexión. Recibido el 29 de agosto de 2018 y aceptado para su publicación el 10 de junio de 2019.

** Docente en el Instituto Superior Tecnológico Bolivariano de Tecnología, Ecuador. (odettemartinezperez@gmail. com) orcid.org/0000-0001-6295-2216
} 


\section{SUMARIO}

1. Reflexiones iniciales

2. El contrato de trabajo en el ámbito del derecho internacional privado: ¿por qué es una garantia?

3. El contrato de trabajo internacional en el ordenamiento jurídico cubano

4. Propuestas dirigidas al perfeccionamiento de las normas de ordenación de la contratación internacional laboral

5. Reflexiones finales

\section{Reflexiones iniciales}

Los cambios auspiciados por la tecnología de la información, la globalización financiera y comercial y la intensificación de procesos regionales de integración económica, particularmente en la Unión Europea, tienen consecuencias sobre toda la economía mundial, en general, y sobre las relaciones laborales, en particular. Las más importantes son el grado de apertura exterior de la economía, el nivel de competencia de los mercados de bienes y servicios, el régimen de la política monetaria, el proceso de ajuste macroeconómico ante perturbaciones de oferta y de demanda, el ritmo de innovación y de desarrollo tecnológico, la capacidad competitiva de las naciones y la heterogeneidad de las empresas y de la fuerza de trabajo. En última instancia, esto condiciona la cuantía y la composición de la demanda de trabajo.

Desde esta perspectiva, el progreso tecnológico manifiesta cada vez más un carácter sesgado a favor de la cualificación de los trabajadores. En los países desarrollados se asiste a un declive del empleo en el sector industrial, un deterioro de las condiciones de trabajo, una posible caída de los salarios reales y la persistencia, todavía hoy en muchos de estos países, de una tasa de desempleo elevada. El resultado es que aumenta la demanda relativa de trabajo cualificado con respecto al no cualificado. Ante este cambio en la composición de la demanda, 128 se observa un incremento de la desigualdad salarial en los países desarrollados a favor de los trabajadores cualificados, y una concentración del desempleo en los segmentos de trabajadores de menor cualificación.

En ese contexto, otra de las tendencias ha sido la rigidez institucional entendida como la existencia de normas que dificultan competir con otros países que no tienen tales normas, o sea, aquellos donde las instituciones laborales y las normas son más laxas o simplemente no existen. En materia laboral, el conflicto se visualiza en el caso de los países receptores en la protección que los Estados puedan dispensar a los trabajadores migrantes, de las acciones de 
los privados y flexibilizar sus propias restricciones de entrada y regularización, sin afectar los índices demográficos vinculados con el empleo. En el caso de los países emisores, no deben verse afectados por el robo de cerebros, a la par que los individuos tienen derecho a buscar mejores ofertas laborales.

De ahí que resulte trascendente la articulación del binomio migracióntrabajo, en una política en favor de los derechos humanos. Cuba no escapa a la realidad que enfrentan los países emisores de migrantes. El sistema de normas de derecho internacional privado en materia laboral en Cuba se encuentra integrado por un conjunto de normas externas e internas, que poseen insuficiencias: lagunas, la indebida construcción de los supuestos de hechos en la norma conflictual, la dispersión legislativa y la inaplicabilidad de muchas normas por obsolescencia de los conceptos jurídicos que presentaban en sus supuestos de hecho y las contradicciones entre las normas convencionales e internas. Lo anterior provoca poca flexibilidad para la recepción de la migración y la imposibilidad de que los migrantes circulares puedan acumular algún tipo de derecho laboral en Cuba, previa contribución monetaria, en una simbiosis entre el sistema tributario y el sistema laboral. Esto se determinó en el estudio exploratorio, que nos animó y reafırmó la necesidad de realizar la investigación en este campo.

\section{El contrato de trabajo en el ámbito del derecho internacional privado: ¿por qué es una garantía?}

Uno de los grandes problemas de la migración laboral en la actualidad son las condiciones de informalidad. Por tanto, atendiendo a que las garantías, como mecanismos jurídicos y extrajurídicos, permiten el reconocimiento y la eficacia de los derechos, el contrato de trabajo se vuelve una figura para proteger al trabajador, ya que legaliza su vínculo y le da un estatus jurídico y, consecuentemente, establece derechos, deberes y garantías para el empleador y el empleado.

El contrato de trabajo como forma de materializar la relación jurídica laboral 129 es "el acuerdo entre el trabajador y la dirección de la organización empresarial, para la formalización de la relación laboral, salvo en los casos en que ésta se establece por designación y elección. Mediante el Contrato de Trabajo, el trabajador y la dirección de la organización empresarial se comprometen a cumplir los deberes, derechos y obligaciones contenidas en el mismo". ${ }^{1}$

\footnotetext{
1 Viamontes Guilbeaux, Eulalia, Derecho Laboral Cubano. Teoría y Legislación, La Habana, Editorial Félix Varela, 2001, pp. 156 y 157.
} 
Se determina la internacionalidad de un contrato por su vínculo con la pluralidad de ordenamientos jurídicos. ${ }^{2}$ Como características principales, dicho instrumento posee un carácter histórico; su regulación ha estado condicionado en diferentes épocas por el flujo migratorio, la tipología de los Estados, la religión, la cultura, la economía y, en el último lustro, por la presión de determinados organismos, como la Organización de Naciones Unidas (oNU), la Organización Internacional del Trabajo (огт) y la Organización Internacional para las Migraciones (огм).

El régimen jurídico del contrato de trabajo internacional consistente en la ley que regula la relación, puede ser la elegida por las partes (para aquellos que admiten la autonomía de la voluntad), o bien por mandato de la norma de conflicto. Pero sí es cierto que, en un caso u otro, esta ley rige para toda la relación contractual, de principio a fin. Por lo tanto, suele denominarse ley aplicable al fondo del asunto, y también se identifica con los latinismos lex causae, ley declarada aplicable a la relación jurídica y lex contractus, simplemente ley del contrato. ${ }^{3}$

A diferencia del contrato de trabajo interno, es práctica común en las relaciones laborales internacionales, y en el contrato de trabajo internacional, que se manifieste el principio de autonomía de la voluntad. Además, el contrato de trabajo internacional hereda la ruptura del principio de unidad contractual por el de fragmentación del contrato.

Las regularidades teóricas del contrato de trabajo internacional que los distinguen del contrato de trabajo interno son las siguientes.

En primer lugar, los sujetos son el trabajador y el empleador. En caso de que el elemento personal marque el carácter internacional, uno de ellos tendrá nacionalidad diferente. Es así que su nacionalidad determinará la ley aplicable a la capacidad y a la representación.

En el caso de los sujetos, una cuestión importante para evaluar en el contrato de trabajo internacional es la capacidad de las partes.

La capacidad que interesa al contrato de trabajo es aquella que permite concertar dicho contrato con eficacia jurídica: la capacidad de obrar, que delimita los requisitos que ha de reunir una persona para que el derecho le permita celebrar un contrato de trabajo válido. Para esto, se exige la concurrencia de determinados requisitos: la aptitud, el cumplimiento de una edad determinada así como la ausencia de causas de incapacitación. ${ }^{4}$

\footnotetext{
2 Lorenzo Idiarte, Gonzalo, "Cuándo un contrato es internacional", en Jan Kleinheisterkamp (coord.), Avances del Derecho Internacional Privado en América Latina, España, Fundación de Cultura Universitaria, 2002, pp. 106-116.

${ }^{3}$ Dávalos Fernández, Rodolfo, Derecho Internacional Privado, La Habana, Universidad de la Habana, 1990, p. 139.

${ }^{4}$ Viamontes Gulbeaux, Eulala, Derecho Laboral Cubano. Teoría y Legislación, La Habana, Editorial Félix Varela, 2001, p. 285.
} 
La edad es un factor imprescindible para determinar la capacidad y no puede obviarse al concertar y cumplir un contrato de trabajo. Por este motivo, cada legislación delimita la exigencia de una edad mínima, por debajo de la cual no se puede contratar válidamente.

La capacidad jurídica laboral del trabajador es más que el cumplimiento de la edad establecida por ley. La relación jurídica laboral no existe si no se concreta en un vínculo específico. Por tanto, la persona natural tiene la capacidad jurídica cuando cumple los requisitos ${ }^{5}$ que se exigen para cada ocupación. ${ }^{6}$

La ley personal regulará la ley aplicable a la capacidad del trabajador como persona física teniendo en cuenta su ciudadanía, es decir, la ley del Estado del que es ciudadano. Por otro lado, la capacidad contractual del empresario ${ }^{7}$ es la capacidad negocial para celebrar contratos de trabajo y se rige por las reglas inherentes a las personas jurídicas. ${ }^{8}$

Uno de los elementos que trae aparejado el factor capacidad es la personalidad jurídica de la entidad laboral, la cual pende de la validez de constitución del ente social. Esto es, se rige por lo que estipule la ley, sus estatutos y reglamentos, ya que su personalidad jurídica nace precisamente con la constitución de esta, sea por resolución, por ley o por los estatutos de la misma. ${ }^{9}$

Es necesario agregar que la celebración de contratos de trabajo por parte de los representantes de la persona jurídica que exceda los poderes conferidos por sus estatutos o escritura fundacional traería consigo la nulidad de dicho contrato. Para actuar, la persona jurídica debe mantener el marco que la ley le ha conferido.

La ley aplicable a las personas jurídicas es la ley de su nacionalidad, ya que no puede descomponerse la ley de acuerdo con la ciudadanía de cada miembro de la entidad. En este caso, como equivalente de la ley nacional, se utiliza la ley del Estado que crea o reconoce a dicha persona jurídica. ${ }^{10}$

La capacidad de la persona jurídica extranjera se rige en correspondencia con la ley de su constitución en cuanto a los derechos y responsabilidades de la sociedad. Sin embargo, es necesario determinar que, en relación con la capacidad de obrar, esta persona jurídica tiene que cumplir con los requisitos

\footnotetext{
${ }^{5}$ Entre estos requisitos se destacan el grado de escolaridad, esencial para obtener la capacidad jurídica laboral; el sexo, que tiene como objetivo fundamental proteger la salud de la mujer; y el estado de salud, ya que las personas discapacitadas no pueden desarrollar todo tipo de actividad laboral.

${ }^{6}$ Viamontes Gullbeaux, Eulala, Derecho Laboral Cubano. Teoría y Legislación, La Habana, Editorial Félix Varela, 2001, p. 104.

7 "Para el Derecho Laboral es empresario, sujeto del Contrato de Trabajo, cualquier persona, ya sea física o jurídica, que hace suyo el resultado o frutos del trabajo, contractualmente prestado, obligándose a remunerarlo."

${ }^{8}$ Viamontes Gulbeaux, Eulalia, Derecho Laboral Cubano. Teoría y Legislación, La Habana, Editorial Félix Varela, 2001, p. 290.

${ }^{9}$ Dávalos Fernández, Rodolfo, Derecho Internacional Privado, La Habana, Universidad de la Habana, 1990, p. 101.

${ }^{10}$ Echemendia, José Miguel, Derecho Internacional Privado II, Habana, Imprenta Ius, 1999, p. 89.
} 
estipulados en el territorio donde desarrolla sus actividades, de acuerdo con las limitaciones que al respecto puedan imponer las leyes del ordenamiento jurídico interno de cada país. ${ }^{11}$

En segundo lugar, la internacionalidad podrá determinarse por el lugar de ejecución del contrato y va a determinar la ley aplicable al contenido, con el principio lex loci laboris. Asimismo, el lugar también determina las formalidades para la concertación del contrato, lex locus regit actum.

La forma del contrato es un elemento que tratan varios autores, quienes han dado un concepto de este término teniendo en cuenta las características de sus sistemas y ordenamientos jurídicos. "Se llama forma del contrato al medio utilizado por las partes para expresar o exteriorizar su consentimiento $\mathrm{y}$, eventualmente, dejar constancia de la materia o estipulaciones sobre las que este recae." 12

Según Eulalia Viamontes, la forma del contrato "es el conjunto de prescripciones que la ley establece referente a las condiciones y términos, expresando que deben observarse en la formación del acto jurídico para su plena validez". ${ }^{13}$

Por su parte, Giuliano y Lagarde manifiestan al respecto que "la forma comprende todo comportamiento exterior y tangible impuesto al autor de una manifestación de voluntad jurídica, sin la cual esta manifestación no se verá atribuida de total eficacia". ${ }^{14}$

Históricamente, el derecho internacional privado sometió la forma de los actos jurídicos, o sea, la validez formal de los actos, al principio locus regit actum. Por lo tanto, es posible afirmar que la regla (el lugar rige el acto) predomina en casi todos los sistemas para determinar las formas y solemnidades de los contratos y otros actos jurídicos. Este principio regula que cualquier acto o negocio jurídico deberá ser reglamentado en su forma y formalidades por la ley del lugar donde se otorgue. ${ }^{15}$

De esta forma, las normas que regulan la validez formal de los contratos quedan dispuestas dentro de la legislación laboral interna de cada país, donde tanto la entidad contratante como el personal contratado se rigen por la legislación imperante. Aplica la ley del lugar donde se suscribe el acto.

En cuanto al contenido, se atiende al lugar de ejecución del contrato y a los diferentes tipos de normas que se vinculan con el mismo. En ese sentido,

\footnotetext{
${ }^{11}$ Dávalos Fernández, Rodolfo, Derecho Internacional Privado, La Habana, Universidad de la Habana, 1990, p. 143.

${ }^{12}$ Montoya Melgar, Alfredo, Derecho del Trabajo, Madrid, Tecnos, 1991, p. 295.

${ }^{13}$ Viamontes Gulbeaux, Eulalla, Derecho Laboral Cubano. Teoría y Legislación, La Habana, Editorial Félix Varela, 2001, p. 158.

${ }^{14}$ Giulano, Manuel y Lagarde, Patricio, Informe referente al Convenio sobre la Ley aplicable a las obligaciones contractuales, La Habana, Editorial DocE, 1992, p. 27.

${ }^{15}$ Dávalos Fernández, Rodolfo, Derecho Internacional Privado, La Habana, Universidad de la Habana, 1990, p. 143.
} 
la autonomía de la voluntad no se puede obviar, dada su importancia dentro de los elementos que conforman el contrato.

Este componente no se establece arbitrariamente. Es la propia ley quien lo regula. Acorde con estas facultades, la autonomía de la voluntad quedará dentro o fuera de la legislación de cada Estado, dependiendo de sus intereses en las esferas económica, política y social. En materia de contratación, constituye un factor fundamental, ya que las partes que hacen uso de dicho principio pueden acordar los aspectos que van a quedar regulados dentro del estatuto contractual.

Según Rodolfo Dávalos, el contenido es "la facultad legal que tienen las partes de elegir el ordenamiento jurídico estatal aplicable a un contrato internacional". ${ }^{16}$ Esta tiene como fin suplir la ausencia de regulación legal adecuada, colmar las lagunas que existan en el contenido de los contratos o, sencillamente, coadyuvar a resolver las dudas que surjan en la interpretación de estos. ${ }^{17}$

En los últimos tiempos ha existido la tendencia a concebir la fuerza de trabajo como mercancía, aplicando la autonomía de la libertad en el contrato internacional de trabajo. Esta postura es criticable cuando se usa de forma absoluta.

Sí es cierto que esta puede utilizarse, porque la internacionalidad de la relación y la conciliación de intereses no ocurre con la presencia de la norma de una sola parte. La autonomía de la voluntad es un factor que no actúa $e x$ lege, fuera del alcance de toda ley; por el contrario, la designación de la ley aplicable por las partes se realiza bajo el supuesto previsto por la ley del país del que se trate y por el sistema de normas del derecho internacional privado bajo el cual toma vida el contrato. ${ }^{18}$

Así, a la hora de establecer el derecho aplicable, las partes no se sitúan fuera del marco legal, sino que efectúan esta selección en función de una ley que las autoriza a ello y que, por lo general, establece también la forma de suplir el olvido mediante una presunción legal de voluntad. Una vez perfeccionado el contrato, las partes no solo quedan obligadas a cumplir lo pactado, sino también a asumir las consecuencias de cualquier incumplimiento.

Desde el punto de vista del derecho aplicable, es necesario apuntar que todo contrato en el que estén presentes elementos extranacionales podrá regirse por la ley que escojan las partes producto a la autonomía de la voluntad, dentro del marco legal de las normas imperativas del lugar de su concertación.

\footnotetext{
${ }^{16}$ Dajalos Fernández, Rodolfo, Peña Lorenzo, Taydit, Santibañez Freire, María del Carmen, Derecho Internacional Privado, La Habana, Félix Varela, 2007, p. 120.

${ }_{17}$ Uria, Rodrigo, Derecho Mercantil, Madrid, Marcial Pons, 1997, p. 22.

${ }^{18}$ Dávalos Fernández, Rodolfo, Derecho Internacional Privado, La Habana, Universidad de la Habana, 1990, p. 15.
} 
Sin embargo, puede existir un concurso de leyes aplicables en el sentido de una vinculación necesaria de la ley escogida con la lex loci laboris. ${ }^{19}$ Es decir, la propia ley del lugar donde se realiza la contratación (donde se efectúa el trabajo) es la que determina si se puede utilizar una ley ajena al ordenamiento jurídico. Dicha norma, dentro de su articulado, especifica esta posibilidad.

La legislación interna de cada país es la que decide el campo de acción de la autonomía de la voluntad. Así, deja a los contratantes aquellos tipos legales que estime pertinentes, para que puedan decidir la ley aplicable, y reserva para sí los que considere necesarios, confiriéndole una tutela jurídica especial. ${ }^{20}$

Pese a la presencia del principio de autonomía de la voluntad, en el ámbito de la contratación, existen determinados países donde las regulaciones estatales definen imperativamente las condiciones en que se realizan las relaciones jurídicas, así como sus requisitos, condiciones y hasta los trámites para obtener su aprobación. Muchos son los ordenamientos jurídicos que crean normas especialmente elaboradas para la contratación internacional. Estos regulan aspectos como la importación, exportación e inversiones, con la finalidad de delimitar las condiciones a las que han de someterse las operaciones entre nacionales y extranjeros en un país determinado. ${ }^{21}$

Las disposiciones imperativas, de acuerdo con el criterio expresado por Espinar Vicente, son "aquellas normas que determinan conminatoriamente las condiciones a las que han de ajustarse todos los actos que durante el desarrollo de la relación jurídica con elemento extranjero hayan de realizarse en el territorio de un Estado cuyo ordenamiento jurídico no sea la lex contractus". ${ }^{22}$

El clásico principio de autonomía de la voluntad también se puede ver limitado, ya que la legislación interna de cada país reserva para sí aquellos tipos legales que dentro de la relación contractual crea necesario concederle especial tutela jurídica.

Por ello, en el caso de la contratación laboral, este principio se restringe en algunos aspectos, ya que con frecuencia se obliga a las partes a realizar operaciones con arreglo a formas rígidamente preestablecidas. Estas limitaciones traen consigo la imposibilidad de discutir el contrato en un plano de igualdad y absoluta libertad para contratar, motivado por una posición dominante en

\footnotetext{
${ }^{19}$ Dávalos Fernández, Rodolfo, Peña Lorenzo, Taydit, y Santibánez Freire, Maria del Carmen, Derecho Internacional Privado, La Habana, Félix Varela, 2007, p.187.

${ }^{20}$ Dávalos Fernández, Rodolfo, Derecho Internacional Privado, La Habana, Universidad de la Habana, 1990, pp. 15-16.

${ }^{21}$ Dávalos Fernández, Rodolfo, Derecho Internacional Privado, La Habana, Universidad de la Habana, 1990, p. 27.

${ }^{22}$ Espinar VICENTE, José MARiA, La regulación jurídica de los contratos internacionales de contenido económico, Madrid, Editoriales de Derecho Reunidas, 1979, p. 111.
} 
el contrato que puede sujetarse a formas predeterminadas por condiciones generales de contratación o contratos tipos rígidos.

Es necesario aclarar que la ley escogida por las partes no puede ser aplicada a todos los elementos del contrato, ya que existen determinados tipos legales que el Estado reserva para sí de forma especial. Entre dichos tipos destacan la capacidad de las partes, que se rige por la ley personal de los contratantes; la forma y la validez formal de los actos, que tienen como presupuesto el principio locus regit actum; y las cuestiones de orden público, ya que cada Estado determina los asuntos que lo afectan dentro de su territorio.

De ahí que el régimen jurídico del contrato esté compuesto por los siguientes elementos: normas constitucionales de orden público internacional; las normas imperativas, fundamentalmente administrativas, en materia migratoria y las regulaciones laborales internas, las normas conflictos de derecho laboral internacional $\mathrm{y}$, finalmente, las normas de derecho internacional público que protegen al trabajador. Esas normativas se incorporan como contenido del contrato de trabajo internacional o se tienen en cuenta para no contravenirlas.

Partiendo de la revisión bibliográfica, Cáceres Serrano y Martínez Pérez ${ }^{23}$ establecieron todo el maniqueísmo funcional del contrato de trabajo internacional. Construyeron reglas para la adecuada construcción de la norma conflicto para el contrato de trabajo laboral internacional, como garantía del migrante:

- Todo Estado debe poseer su Código de Derecho Internacional Privado y un capítulo de derecho laboral internacional, con una sección donde se plasmen las normas de contratación laboral internacional. En caso contrario, en el Código de Trabajo debe existir un capítulo con las normas al respecto.

- Debe quedar definido el contrato de trabajo internacional en el Código de Trabajo interno de cada país o en un Código de Derecho Internacional Privado.

- Debe considerarse al empleador extranjero y la persona natural extranjera, como sujetos que dotan de internacionalidad al contrato.

- Debe quedar expuesto el principio de condición de internacionalidad por el lugar de concertación o de ejecución del contrato.

- Debe consagrarse un reenvío a los principios constitucionales garantes de extranjería y a la legislación migratoria y de inversión extranjera

${ }^{23}$ Martinez Pérez, Odeme y Serrano Cáceres, Yanara, Premisas para la regulación normativa del contrato de trabajo internacional en Cuba, Tesis de maestría en opción al grado de Doctora en Ciencias Jurídicas, Cuba, Facultad de Derecho Universidad de Oriente, inédita. 
correspondiente, así como a las normas del Código de Trabajo expresamente aplicables a la relación y a cualquier otra norma imperativa.

- Exponer los requisitos de fondo y procedimientos necesarios, para definir la capacidad y representación de los empleadores.

- La capacidad de los trabajadores extranjeros, junto a un reenvío y prohibición de transgredir su estatuto personal.

- Debe consagrarse el principio de lex loci laboris y el de lex locus regit actum.

- Determinación del valor y reenvío específico a las normativas internacionales aplicables.

- Reenvío a las normas generales de solución de dificultades y limitaciones en la aplicación del derecho extranjero.

- En busca de preservar la coherencia y unidad del ordenamiento jurídico en su formulación, debe proveerse del uso correcto del lenguaje jurídico, elaborar actos normativos simples que sean accesibles en su comprensión y evitar los términos ambiguos, contradicciones, lagunas, vaguedades y reiteraciones, sobre todo durante el establecimiento de los sujetos, en las normas que establecen derechos, deberes y garantías.

- El método bilateral o multilateral debe ser usado en aquellas normas conflicto del sistema conflictual tradicional señaladas, a través de un procedimiento que puede sistematizarse de la siguiente manera: con relación al supuesto de hecho de las mismas, se debe atender a cada una de las variantes específicas en las que puede diseccionarse cada instituto. En relación con este problema, el legislador puede realizar una subdivisión del supuesto de hecho. Es decir, puede desglosarlo en cada una de sus distintas variantes y establecer un precepto de atribución diferente para cada una de las esferas de regulación señaladas.

- En el caso del precepto de atribución, la consecuencia jurídica puede utilizar conexiones sucesivas jerárquicamente ordenadas. Este modo de formular las conexiones permite tomar en consideración las diversas maneras en las que puede producirse, a nivel concreto, lo abstractamente descrito en el presupuesto de la norma. Para ello, pueden observarse dos notas definitorias con los puntos de conexión, ciudadanía y residencia. A cada una de las esferas anteriores le corresponde un determinado punto de conexión y no otro.

- Referente al conflicto móvil, no debe ser objeto de una solución general, sino que la norma debe prescribir su solución en atención a las peculiaridades del caso. Para ello debería tomarse la solución alternativa, consistente en aplicar una u otra ley en atención a criterios de validación del acto o de protección de los intereses en juego. 
De este análisis, es posible determinar que la ley declarada aplicable al contrato, fuera del ejercicio de la autonomía de la voluntad y pese a la existencia del principio de unidad, no habrá de regir por sí sola todo el supuesto de la contratación. Antes bien, alrededor de la relación jurídica se dará un concurso de leyes aplicables que van desde las acordadas por las partes, lex causae, hasta las normas imperativas del foro, lex fori.

\section{El contrato de trabajo internacional en el ordenamiento jurídico cubano}

Teniendo en cuenta el criterio de las autoras mencionadas, puede definirse que la tendencia histórica en Cuba es que la internacionalidad se mueva en función del sujeto, como objeto de regulación de la norma.

En este último período, se observan las principales insuficiencias de las normas actuales:

- No existe un código actual de derecho internacional privado, a partir del hecho de que las normas conflicto en Cuba se encuentran en el Código Civil y las leyes especiales. En este caso, el Código de Trabajo no regula el contrato de trabajo internacional.

- No queda expuesto con claridad el principio de condición de internacionalidad por el lugar de concertación o de ejecución del contrato, para que así alcance en su protección a los nacionales, incluso a los que gestionan su contratación laboral en el exterior, siempre que la norma conflicto extranjera dé paso a la ley de nuestro foro.

- No se consagran los principios de lex loci laboris y el de lex locus regit actum.

- No se determina el valor y reenvío específico a las normativas internacionales aplicables y la jerarquía con respecto al Código de Trabajo, a partir de las dificultades que en este aspecto comporta la norma cubana.

- No se determinan las normas generales de solución de dificultades y limitaciones en la aplicación del derecho extranjero del Código Civil.

De manera especial, la regulación jurídica de la contratación de servicios profesionales de cubanos en el exterior se caracteriza por:

- Materializar los principios de territorialidad, reciprocidad y lex loci laboris.

- Ser dispersa, anómica y con vaguedades. 
- Carecer de un mecanismo de control de orden público, internacional e interno de los derechos, deberes y garantías de los trabajadores y los intereses estatales, donde se contrasta su conformidad con la Constitución de la República, los tratados en materia laboral suscritos por Cuba y la legislación laboral vigente.

- Impedir que, con el nuevo sistema de migración circular que se ha implementado con la reforma migratoria, aquellos cubanos que trabajan en el exterior y gestionan contratos personales, durante dos años, entren al sistema laboral y tributario, y tanto el país como los trabajadores se beneficie económicamente de ello.

Por lo anterior, y atendiendo el criterio de las autoras, en la investigación citada se hace necesario reformular las normas en materia del derecho internacional privado y, especialmente, en materia de contrato laboral internacional.

\section{Propuestas dirigidas al perfeccionamiento de las normas de ordenación de la contratación internacional laboral}

Debido a las actuales condiciones de la emigración, cuyas características son la circularidad, el saldo negativo, el robo de cerebros y el flujo emigratorio de personal calificado, la actual norma es inoperante. Dichas limitaciones no incluyen ni protegen a los migrantes circulares que gestionan contratos de trabajo en el exterior. Además, le permiten al Estado aprovechar económicamente esa fuerza profesional formada en Cuba.

Por otra parte, las políticas de incentivo al turismo, la inversión extranjera y el incremento de población extranjera en Cuba evidencian la necesidad de perfeccionar estas normas atendiendo a los principios políticos, económicos y sociales contenidos en la actual Constitución de la República, los tratados firmados por Cuba, especialmente el de la protección de los trabajadores migratorios, y el actual Código de Trabajo.

En este sentido, mi propuesta inicial se dirige a la adopción de una ley de derecho internacional privado, donde se defina como contrato de trabajo internacional aquel que se materializa cuando confluyen elementos de carácter extranacional dados por el sujeto (dígase trabajador o empleador), el objeto o el lugar donde se lleva a vías de hecho el contrato, con la intervención de una o más conexiones extranjeras o pluralidad de ordenamientos jurídicos. Interesa al derecho internacional privado por no estar ligado exclusivamente al derecho interno de un país en específico. 
Asimismo, sugiero que se defina con claridad que quedan autorizados para trabajar en Cuba los extranjeros, según se ha establecido en la Ley de Extranjería, con las clasificaciones de residentes temporales y residentes permanentes. La capacidad laboral de los trabajadores extranjeros y cubanos que laboren en el exterior atenderán a lo dispuesto en el Código de Trabajo de la República de Cuba, siempre que no entren en conflicto con su estatuto personal. De ser así, se atenderá a este último.

Se debe regular la obediencia de los contratos realizados en Cuba por trabajadores extranjeros, a la normativa constitucional, si su ejecución es en la isla, en respeto al orden constitucional y a que las formalidades de los contratos internacionales se regirán por la ley del lugar donde sean realizados.

Con respecto al contenido de los contratos de trabajo internacional que se ejecuten en Cuba, se atenderá a lo previsto en la ley cubana, y los que sean firmados para su ejecución en el exterior atenderán a la ley del lugar de su ejecución o los convenios firmados por la República. Asimismo, para el tratamiento de las dificultades y limitaciones en la aplicación del derecho extranjero que se presenten alrededor de estos se atenderá a lo previsto en el Código Civil en su carácter supletorio.

\section{Reflexiones finales}

La regulación jurídica cubana, para la contratación de servicios profesionales en el exterior se caracteriza por tres elementos: $a$ ) Materializa los principios de territorialidad, reciprocidad y lex loci laboris; b) Se encuentra dispersa, anómica y existen vaguedades; $y, c)$ Carece de un mecanismo de control de los derechos, deberes y garantías de los trabajadores y los intereses estatales.

Entre las principales insuficiencias en su contenido se encuentran las siguientes:

- La dispersión en la legislación y atención diferenciada en la materia pudiera ser objeto de un tratamiento dispar para los diferentes trabajadores que se subsumen a un régimen jurídico u otro, lo que resulta en una vulneración al principio de igualdad en la arista laboral.

- No permite que, con el nuevo sistema de migración circular que se ha implementado en el país, aquellos cubanos que trabajan en el exterior, por gestión personal y no han perdido la residencia, entren al sistema laboral y el país se beneficie económicamente de ello y los trabajadores también.

- Son restringidos los sujetos destinatarios de la misma que pudieran ingresar al Estado y ser protegidos por esta resolución, "todos los cubanos 
cuya motivación de viaje sea laboral en una empresa extranjera, siempre y cuando la última no haya contravenido en su historial empresarial los principios económicos, políticos y sociales del país”.

Entre sus ventajas pueden señalarse la existencia de las condiciones generales de contratación, tanto del contrato de trabajo individual, como el de suministro de fuerza de trabajo. No obstante, pudiera señalarse como negativo que estas modificaciones tienen una formulación de principios y las regulaciones jurídicas o proformas utilizadas por las agencias empleadoras. En ocasiones no se ajustan a los principios previstos en la Constitución de la República, los convenios suscritos por Cuba y la legislación laboral vigente.

\section{Bibliografía}

DÁvalos Fernández, Rodolfo, Derecho Internacional Privado, La Habana, Universidad de la Habana, 1990.

Dávalos Fernández, Rodolfo, Peña Lorenzo, Taydit, Santibáñez Freire, María del Carmen, Derecho Internacional Privado, La Habana, Félix Varela, 2007.

ESPINAR VICENTE, José MARÍA, La regulación jurídica de los contratos internacionales de contenido económico, Madrid, Editoriales de Derecho Reunidas, 1979.

Echemendía, José Miguel, Derecho Internacional Privado II, Habana, Imprenta IUS, 1999 Giuliano, Manuel y Lagarde, Patricio, Informe referente al Convenio sobre la Ley aplicable a las obligaciones contractuales, La Habana, Editorial DocE, 1992.

LoRenzo Idiarte, Gonzalo, “Cuándo un contrato es internacional”, en Jan Kleinheisterkamp (coord.), Avances del Derecho Internacional Privado en América Latina, España, Fundación de Cultura Universitaria, 2002.

Martínez Pérez, Odette y Serrano Cáceres, Yanara, Premisas para la regulación normativa del contrato de trabajo internacional en Cuba, Tesis de maestría en opción al grado de Doctora en Ciencias Jurídicas, Cuba, Facultad de Derecho Universidad de Oriente, inédita.

Montoya Melgar, Alfredo, Derecho del Trabajo, Madrid, Tecnos, 1991.

140 URíA, Rodrigo, Derecho Mercantil, Madrid, Marcial Pons, 1997.

Viamontes Guilbeaux, Eulalia, Derecho Laboral Cubano. Teoría y Legislación, La Habana, Editorial Félix Varela, 2001. 